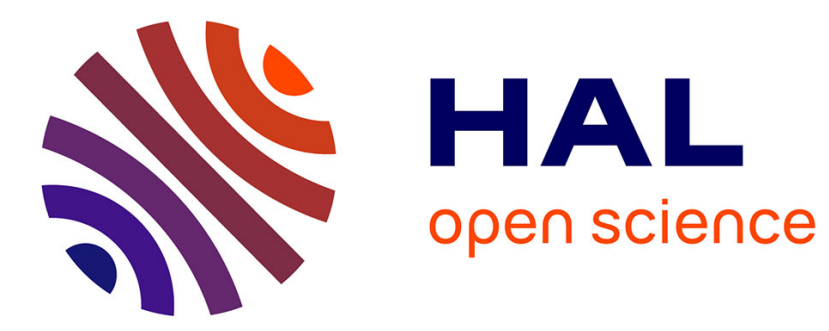

\title{
The cool surfaces of binary near-Earth asteroids
}

\author{
Marco Delbo, Kevin Walsh, Michael Mueller, Alan W. Harris, Ellen S. Howell
}

\section{To cite this version:}

Marco Delbo, Kevin Walsh, Michael Mueller, Alan W. Harris, Ellen S. Howell. The cool surfaces of binary near-Earth asteroids. Icarus, 2011, 212 (1), pp.138. 10.1016/j.icarus.2010.12.011 . hal00725402

\section{HAL Id: hal-00725402 \\ https://hal.science/hal-00725402}

Submitted on 26 Aug 2012

HAL is a multi-disciplinary open access archive for the deposit and dissemination of scientific research documents, whether they are published or not. The documents may come from teaching and research institutions in France or abroad, or from public or private research centers.
L'archive ouverte pluridisciplinaire HAL, est destinée au dépôt et à la diffusion de documents scientifiques de niveau recherche, publiés ou non, émanant des établissements d'enseignement et de recherche français ou étrangers, des laboratoires publics ou privés. 


\section{Accepted Manuscript}

The cool surfaces of binary near-Earth asteroids

Marco Delbo, Kevin Walsh, Michael Mueller, Alan W. Harris, Ellen S. Howell

PII:

S0019-1035(10)00468-9

DOI:

10.1016/j.icarus.2010.12.011

Reference:

YICAR 9662

To appear in:

Icarus

Received Date:

8 March 2009

Revised Date:

21 October 2010

Accepted Date:

8 December 2010

Please cite this article as: Delbo, M., Walsh, K., Mueller, M., Harris, A.W., Howell, E.S., The cool surfaces of binary near-Earth asteroids, Icarus (2010), doi: 10.1016/j.icarus.2010.12.011

This is a PDF file of an unedited manuscript that has been accepted for publication. As a service to our customers we are providing this early version of the manuscript. The manuscript will undergo copyediting, typesetting, and review of the resulting proof before it is published in its final form. Please note that during the production process errors may be discovered which could affect the content, and all legal disclaimers that apply to the journal pertain. 


\title{
The cool surfaces of binary near-Earth
}

$$
\text { asteroids * }
$$

\author{
Marco Delbo' a,*, Kevin Walsh ${ }^{\text {a }}$, Michael Mueller ${ }^{\mathrm{a}, \mathrm{b}}$, \\ Alan W. Harris ${ }^{c}$, Ellen S. Howell ${ }^{\mathrm{d}}$ \\ a UNS-CNRS-Observatoire de la Côte d'Azur, Laboratoire Cassiopée, BP 4229 \\ 06304 Nice cedex 04, France. \\ ${ }^{\mathrm{b}}$ Steward Observatory, University of Arizona, $933 \mathrm{~N}$ Cherry Ave, Tucson AZ \\ 85721, USA. \\ ${ }^{\mathrm{c}}$ DLR Institute of Planetary Research, Rutherfordstrasse 2, 12489 Berlin, \\ Germany. \\ dArecibo Observatory, HC3 Box 53995, Arecibo, PR 00612, USA.
}

Number of pages: 40

Number of tables: 3

Number of figures: 5 
Running head: The cool surfaces of binary near-Earth asteroids.

Editorial correspondence and proofs should be directed to:

Marco Delbo'; delbo@oca.eu; Tel: +33 492001944 


\section{Abstract}

Here we show results from thermal-infrared observations of km-sized binary NearEarth Asteroids (NEAs). We combine previously published thermal properties for NEAs with newly derived values for three binary NEAs. The $\eta$ value derived from the Near-Earth Asteroid Thermal Model (NEATM) for each object is then used to estimate an average thermal inertia for the population of binary NEAs and compared against similar estimates for the population of non-binaries. We find that these objects have, in general, surface temperatures cooler than the average values for non-binary NEAs as suggested by elevated $\eta$ values. We discuss how this may be evidence of higher-than-average surface thermal inertia. This latter physical parameter is a sensitive indicator of the presence or absence of regolith: bodies covered with fine regolith, such as the Earth's Moon, have low thermal inertia, whereas a surface with little or no regolith displays high thermal inertia. Our results are suggestive of a binary formation mechanism capable of altering surface properties, possibly removing regolith: an obvious candidate is the YORP effect.

We present also newly determined sizes and geometric visible albedos derived from thermal infrared observations of three binary NEAs: (5381) Sekhmet, (153591) $2001 \mathrm{SN}_{263}$, and (164121) $2003 \mathrm{YT}_{1}$. The diameters of these asteroids are $1.41 \pm 0.21$ $\mathrm{km}, 1.56 \pm 0.31 \mathrm{~km}$, and $2.63 \pm 0.40 \mathrm{~km}$, respectively. Their albedos are $0.23 \pm 0.13$ $0.24 \pm 0.16$, and $0.048 \pm 0.015$, respectively.

Key words: Asteroids, Satellites of asteroids, Asteroids surfaces, Infrared observations. 


\section{Introduction}

The population of known binary NEAs has grown steadily to more than 30 objects discovered by photometric lightcurves and radar observations (Pravec and Harris, 2007; Richardson and Walsh, 2006; Noll, 2006). Binary systems are uniquely interesting in that the analysis of their mutual orbits enables a determination of the elusive mass density, which in turn allows conclusions to be drawn on the bulk composition and porosity. Further information on their physical properties may be gained from studies of binary formation.

In this context, it is intriguing that the majority of these systems are found with a rapidly rotating primary, with rotation periods between 2.2 and 3.6 h, which are among the most rapid rotations found for NEAs (Pravec et al., 2006). Among all 325 NEAs with a diameter D > $200 \mathrm{~m}$ and known lightcurve period, none are observed to have periods shorter than $2.2 \mathrm{~h}$ (Pravec et al., 2007). For faster spin rates, surface material could reach orbital speeds.

The satellites in these systems are almost always on close orbits, between 2 and 5 primary radii, $R_{\text {pri }}$, and are typically found with low eccentricity, close to zero. The satellites' spin periods, where known, are typically synchronized

\footnotetext{
^ Observations of (164121) $2003 \mathrm{YT}_{1}$ were obtained with the TIMMI2 at the $3.6 \mathrm{~m}$ telescope of the European Southern Observatory (PI G.P. Tozzi). Observations of (153591) $2001 \mathrm{SN}_{263}$ and (5381) Sekhmet were obtained at the Infrared Telescope Facility, which is operated by the University of Hawaii under Cooperative Agreement no. NCC 5-538 with the National Aeronautics and Space Administration, Science Mission Directorate, Planetary Astronomy Program.

* Corresponding author. Email address: delbo@oca.eu (Marco Delbo').
} 
with the orbital periods. Discoveries by lightcurve are biased to only discover satellites larger than $\sim 20 \%$ the size of the primary body, and also are biased against finding them beyond $\sim 10 R_{\text {pri }}$ (Pravec et al., 2006). Radar observations are not similarly biased, and can discover satellites out to much further distances and to much smaller sizes, but have found only a couple of such systems, namely (153591) $2001 \mathrm{SN}_{263}$ and $1998 \mathrm{ST}_{27}$ (Nolan et al., 2008; Becker et al., 2008; Benner et al., 2001). The general characterization of the NEA binary population presented above, with rapidly rotating primaries and close secondaries applies to $\sim 32$ of the 35 known systems.

The similarities in the observed orbital properties of binary NEAs point at a common formation mechanism. It is likely that they formed through spin-up and disruption by the YORP-effect (Yarkovsky O'Keefe Radzievskii Paddack) (Walsh et al., 2008; Scheeres, 2007). The YORP-effect works by the creation of small torques through the reflection and thermal re-emission of solar radiation from an irregularly shaped body (Rubincam, 2000; Rubincam et al., 2002). A substantially non-symmetric body can generate substantial torques (clearly represented in the cartoon in Figs. 1 and 2 in Rubincam 2000), but even smallscale surface features are capable of producing similar torques (Statler, 2009). This effect has been shown to modify spin rates of NEAs (Taylor et al., 2007; Lowry et al., 2007; Kaasalainen et al., 2007) and it can increase or decrease the spin period depending on the spin-pole orientation. The distribution of spin rates among small Main Belt Asteroids (MBAs) and NEAs shows an excess in both fast and slow rotators, which can be produced through the YORP effect acting widely on the populations (Pravec et al., 2008).

Models of YORP-spinup of gravitational aggregates, or "rubble piles", have reproduced the fundamental properties of observed NEA binaries: rapid primary 
rotation, oblate primaries, and basic secondary orbital properties (semi-major axis and eccentricity) (Walsh et al., 2008). This formation mechanism works by slowly pushing bodies towards, and then beyond, their critical spin rates. In the simulations of Walsh et al. (2008), the body takes an oblate shape, which allows ejected mass to accumulate into a satellite in a stable orbit. The simulations show that surface material on the original body is ejected and accumulated into a secondary. In tests where specific surface particles are tracked throughout the simulation, Walsh et al. (2008) found that anywhere from $15-30 \%$ of the surface on the primary is removed, uncovering originally sub-surface material. Those simulations consist of 1000 similar-sized or nearly similar-sized particles, which means each particle represents the large constituent pieces (boulders) of a rubble pile.

It is unclear how fine-grained surface material (regolith), which is not resolved in the Walsh et al. (2008) simulations, responds to large-scale surface mobility. We present three plausible scenarious:

(1) The regolith is shed off the surface before any significant mass (large constituent pieces) is lost. If this is the case, then most rapidly rotating NEAs could have altered surface properties compared to other NEAs. With no satellite or large masses in orbit, it is possible that all regolith ejected off the asteroid's surface would escape from the asteroid via radiation effects on timescales faster than satellite accumulation. Theoretically this could be detected in comparisons of surface properties between all rapidly rotating NEAs against all NEAs.

(2) The regolith moves with the large pieces of the rubble pile. The surface properties change only after binary formation has begun, and significant mass has been lofted into orbit. This may also mean that the total sur- 
face alteration depends on how far into the binary-formation process the system has progressed. This could be manifested in differing surface properties for binary NEAs as compared to the rest of the NEA population, including the subset of rapidly rotating NEAs.

(3) The regolith does not move, and continues to cover the surface of the asteroid although the large constituent pieces of the body move and escape. This scenario would likely lead to no observable changes in the surface properties of the body, except for possibly uncovering younger, less weathered regolith. Potentially this could be detected via spectroscopic signatures of the new, less weathered, regolith. However, the thermal inertia and albedo of the binary systems would likely remain unchanged in comparison to non-binaries due to similar amounts of regolith covering.

Alteration of regolith on asteroids is in principle observable at the wavelengths of their thermal infrared emission; a surface depleted of regolith has a less efficient thermal insulation than one covered with fine and thick regolith. Consequently the former has a higher surface thermal inertia than the latter.

Thermal inertia, $\Gamma$, is a measure of the resistance of a material to changes in surface temperature. It is defined as the square root of the product of the thermal conductivity $(\kappa)$, the heat capacity $(c)$ and the material density $(\rho)$, i.e. $\Gamma=\sqrt{\kappa c \rho}$. Thermal inertia controls the body's diurnal temperature profile and thus affects the intensity and shape of spectral energy distribution of the body's infrared heat emission. For example, in the unrealistic case of zero thermal inertia a body has a prominent temperature peak at the subsolar point and its temperature distribution falls to zero at the terminator.

In the more realistic case of a body with finite thermal inertia and rotating 
with a spin vector not pointing toward the Sun, the temperature distribution is no longer symmetric with respect to the subsolar point. Each surface element behaves like a capacitor or sink for the solar energy such that the body's diurnal temperature profile becomes more smoothed out in longitude (e.g. Spencer et al., 1989; Delbo' and Harris, 2002; Delbo', 2004; Mueller, 2007). The hottest temperature during the day decreases, whereas those on the night-side do not drop to zero as in the idealistic case of zero thermal inertia, implying non-zero thermal-infrared emission from the dark side of the body. So, information about the thermal inertia of an asteroid can be obtained from the color temperature of the body: high thermal inertia causes the day side color temperature of an object to decrease compared to that of another body with a lower thermal inertia.

In this paper we will present new measurements of thermal infrared emission for NEA binaries as well as discuss previously published data. Together the sample is significant enough to identify some basic trends that support significant surface alteration in NEA binaries as compared with other rapidly rotating NEAs and NEAs in general. In section 2 we discuss how the surface thermal inertia of asteroids can be inferred from thermal observations, in section 3 we present new observations, data reduction and analysis via thermal models, in section 4 we derive the thermal inertia of the binary NEAs and we compare it with the value derived for the whole population of NEAs, finally in section 5 we discuss how the observations relate to the YORP model of NEA binary formation. 


\section{Determination of asteroids' thermal inertia}

The thermal inertia of the surface of an asteroid depends on regolith particle size and depth, degree of compaction, and exposure of solid rocks and boulders within the top few centimeters of the subsurface (see e.g. Mellon et al., 2000). Knowledge of the thermal inertia of asteroids is important because its value can be used to infer the presence or absence of loose material on the surface. Lunar regolith, a layer of fragmentary incoherent rocky debris covering the surface of the Moon, has a low thermal inertia of about $\sim 50 J \mathrm{~m}^{-2} \mathrm{~s}^{-0.5} \mathrm{~K}^{-1}$ (see e.g. Winter and Krupp, 1971; Spencer et al., 1989, and references therein). Coarse sand has a higher thermal inertia, about $400 \mathrm{Jm}^{-2} \mathrm{~s}^{-0.5} \mathrm{~K}^{-1}$ (Presley and Christensen, 1997b; Mellon et al., 2000; Christensen et al., 2003, see e.g.), whereas that of bare rock is larger than $2500 \mathrm{Jm}^{-2} \mathrm{~s}^{-0.5} \mathrm{~K}^{-1}$ (Jakosky, 1986), and the thermal inertia of metal rich asteroidal fragments can be larger than 12,000 $\mathrm{Jm}^{-2} \mathrm{~s}^{-0.5} \mathrm{~K}^{-1}$ (Burns et al., 1979, and references therein). The correlation between increasing thermal inertia with increasing regolith grain size is observed in laboratory studies of particulate materials (see e.g. Presley and Christensen, 1997a,b, for a review).

Caution must be exercised when comparing thermal-inertia values between different parts of the Solar System, since thermal inertia is temperature dependent. Heat conduction within regolith (in a vacuum) is dominated by radiative conduction between grains, hence the thermal conductivity is proportional to $T^{3}$, with $T$ being the temperature of the regolith (see e.g. Kührt and Giese, 1989; Jakosky, 1986, and references therein). In this case $\Gamma \propto T^{3 / 2}$ and thus $\Gamma \propto r^{-3 / 4}$, where $r$ is the heliocentric distance. However, this is a negligible effect for our current purpose, since NEAs are observed at very similar helio- 
centric distances around 1 AU. For this reason, the thermal inertia of NEAs can be directly compared to that of the Moon.

As of now, the thermal inertia of some 25 asteroids has been measured (see Delbo' and Tanga, 2009, for a recent overview). It was found (Delbo' et al., 2007; Delbo' and Tanga, 2009) that thermal inertia and object size correlate, in the sense that smaller objects have higher thermal inertia, suggesting a thinner, coarser and less mature regolith and/or a larger abundance of boulders. This is confirmed by in-situ spacecraft observations of the regolith of the NEAs (25143) Itokawa (Yano et al., 2006) and (433) Eros (Veverka et al., 2001a,b); see also Mueller (2007) for the most recent thermal inertia determinations of these objects.

The thermal inertia of an asteroid can be directly derived by comparing measurements of its thermal-infrared emission to synthetic fluxes generated by means of a thermophysical model (TPM; Spencer 1990; Lagerros 1996; Emery et al. 1998; Delbo' 2004; Delbo' et al. 2007; Mueller 2007), which is used to calculate the temperature distribution over the body's surface as a function of the bolometric Bond albedo $(A)$, macroscopic roughness $(\bar{\theta})$, and thermal inertia $(\Gamma)$. The dependence of the thermal infrared flux upon the body's size is trivial: $f(\lambda) \propto D^{2}$ for all values of $\lambda$.

The values of $A, \bar{\theta}, \Gamma$ and $D$ are adjusted until model fluxes best fit the measured ones (see Mueller, 2007, for the description of the method). However, the applicability of TPMs is limited to the few asteroids for which gross shape, rotation period, and spin axis orientation are known. Multi-epoch observations are also required for obtaining a robust TPM fit.

Unfortunately, the large majority of asteroids for which we have thermal in- 
frared observations have been observed at a single epoch and/or information about their gross shape and pole orientation is not available, precluding the use of TPMs. In these cases simpler thermal models such as the near-Earth asteroid thermal model (NEATM; Harris 1998) are used to derive the sizes, albedos and apparent color temperatures of the surface of these objects. The latter can be used to infer the value of the thermal inertia. The NEATM assumes that the object has a spherical shape, and its surface temperature distribution is described by Eqs. (1) and (2):

$$
T(\vartheta)=T_{\max } \cos (\vartheta)^{1 / 4}
$$

where $\vartheta$ is the angular distance of any point on the surface from the sub-solar point. The maximum temperature, reached at the subsolar point, is obtained by the balance between the absorbed radiation (from sunlight) and the emitted radiation:

$$
(1-A) S_{\odot} r^{-2}=\eta \epsilon \sigma T_{\max }^{4}
$$

where $S_{\odot}$ is the solar constant at $1 \mathrm{AU}\left(1329 \mathrm{Wm}^{-2}\right), A$ is the asteroid bolometric Bond's albedo, $r$ is the asteroid heliocentric distance, $\epsilon$ is the infrared emissivity, and $\eta$ is the so-called "beaming parameter". In the NEATM, $\eta$ is adjusted in the fitting procedure to allow the model spectral energy distribution to match the observed data.

The parameter $\eta$ can be seen as a measure of the departure of the asteroid temperature distribution from that of a spherical, smooth body with all surface points in instantaneous thermal equilibrium with sunlight. In particular, thermal inertia causes dayside temperatures to decrease and hence $\eta>1$.

All real surfaces have $\Gamma>0$. However, $\eta$ is not always $>1$, except for perfectly smooth surfaces. Macroscopic roughness, which can be enhanced by the 
presence of craters on the surface, increases the observed temperature of a surface, when the latter is viewed under a small phase angle. The bottom of craters, canyons, valleys are, in general, hotter than the surrounding flat areas, because they are not only illuminated by the Sun, but they also receive scattered sunlight and the thermal infrared emission from the crater walls or the surrounding relief. A rough surface observed at small solar phase angle has $\eta<1$. However, while for a small degree of surface roughness, $\eta$ is smaller than unity and constant with phase angle, for very rough surfaces $\eta$ increases with increasing phase angle (see Delbo', 2004, §6.6.2). Moreover, numerical simulations performed by the same author using a TPM show that $\eta<1.2$ for any degree of roughness and for phase angles up to $90^{\circ}$ assuming zero thermal inertia.

A value of $\eta$ significantly above unity at low or moderate phase angles $\left(<45^{\circ}\right)$ or significantly above $\sim 1.2$ at larger phase angles is an indication that thermal inertia plays a role in decreasing the observed surface temperature. Delbo' et al. (2003) noted that qualitative information about the average thermal inertia of a sample of NEAs could be obtained from the distribution of the $\eta$ values of the sample as a function of the phase angle, $\alpha$. In a subsequent work (Delbo', 2004) it was observed that for a synthetic population of spherical asteroids with constant values of the albedo $(A)$, heliocentric distance $(r)$, thermal inertia $(\Gamma)$, rotation period $(P)$, and surface roughness $(\bar{\theta})$, but with pole directions $\left(\lambda_{0}, \beta_{0}\right)$ randomly oriented, the distribution of the points in the $(\eta, \alpha)$ plane is strongly dependent on the value of $\Gamma$.

If we assume the thermal inertia to be roughly constant within a population of asteroids (e.g. binary NEAs), the distribution of the measured $\eta$-values versus $\alpha$ of different asteroids can be used to infer the thermal inertia of the popula- 
tion. This is the basis of a statistical inversion procedure developed by Delbo' et al. (2007) to determine the mean value of the thermal inertia of NEAs. The method is based on the comparison of the distributions of NEATM $\eta$-values vs $\alpha$ with that of a synthetic population of asteroids generated through a thermophysical model, using realistic distributions of the input parameters $P$, $\lambda_{0}, \beta_{0}$, and $A$ for the whole NEA population derived from the literature. This

approach is based on the fact that, even though shapes, rotation periods, and spin axis orientations are not known for every object, the distribution of these quantities for the whole population can be inferred from published data.

In this work, the method of Delbo' et al. (2007) is applied to binary NEAs. New thermal-infrared observations are presented herein, in addition to $\eta$ values compiled from the literature.

\section{Thermal infrared observations, data reduction and NEATM fit}

In this section we present new thermal infrared observations, the adopted data reduction procedure and the NEATM fit of the binary NEAs (5381) Sekhmet and (164121) $2003 \mathrm{YT}_{1}$, and of the triple NEA (153591) $2001 \mathrm{SN}_{263}$.

\section{(5381) Sekhmet}

(5381) Sekhmet was observed on 2003 May 12-16 UT using the IRTF and the Mid-InfraRed Large-well Imager (MIRLIN; Ressler et al., 1994). A three-point chop-nod pattern was used such that the target was roughly centered in the field of view for $50 \%$ of the time and on two offset positions (North/South) for $25 \%$ of the time each. MIRLIN's N1, N3, and N4 filters were used, with 
effective wavelengths of $8.81,10.27$, and $11.70 \mu \mathrm{m}$. Sekhmet was observable in the second half of the night; those parts of the night were clear on May 12-15. On May 16, observations had to be aborted due to incoming clouds after cycling through the filters once. The humidity was consistently high in all five nights, with $\tau$ measured at the CSO ranging between 0.15 and 0.2 . See Table 1 for observing dates and circumstances.

Images obtained in the four chop-nod beams were subtracted from one another like $(A-B)-(C-D)$, resulting in one 'positive' and two 'negative' detections. After registering the three detections per observation, instrument counts were determined through standard synthetic-aperture photometry routines. Flux calibration was performed against observations of Cohen et al. (1995, 1999) standard stars $\alpha$ Hya and $\gamma$ Aql. Calibration standards and the target asteroid were observed at similar airmass, final fluxes are therefore rather insensitive to airmass correction. We assumed the average extinction coefficients for Mauna Kea reported by Krisciunas et al. (1987): 0.134, 0.109, and $0.095 \mathrm{mag} /$ airmass for $8.81,10.27$, and $11.7 \mu \mathrm{m}$, respectively. Instrument zeropoints were stable at the few- $\%$ level for May 13, 15, and 16, but varied by $\sim 10 \%$ on May 14 . Only one calibration observation per filter was made on May 12. Color corrections were found to be $1 \%$ or smaller and were not applied.

A hardware failure in MIRLIN rendered every eighth readout-row unusable. We replaced those rows with the respective pixel-by-pixel average of the two adjacent rows. The typical FWHM of the point-spread function was around 4 pixels. Some observations were largely unaffected by defective rows while others were severely compromised, with a defective row running through the centroid of one or more detection. In the latter case, we increased the flux uncertainty derived from aperture photometry by up to $100 \%$, weighting the 
detections by the integration time spent on them: $50 \%$ for a severely affected positive detection, $25 \%$ per severely affected negative detection. See Table 2 for final fluxes.

No $H$ value for Sekhmet is available in the published literature. We assumed $H=16.5$ as given by the Minor Planet Center; an uncertainty of 0.5 is assumed. No published lightcurve data are available. Pravec et al. report a "prepublished" lightcurve amplitude of 0.1 mag or larger. ${ }^{1}$ Lightcurve correction of our thermal data was not attempted.

We used the NEATM to fit the thermal fluxes of Sekhmet given in Table 2, separately for each night using the observing geometry given in Table 1 . The zero point for flux calibration was observed to vary by some $10 \%$ on May 14 ; all fluxes obtained in that night were assumed to be uncertain by this factor, dominating over the statistical flux uncertainties reported in Table 2. In order to estimate the statistical uncertainty in the fit parameters $\eta, D$, and $p_{V}$, a Monte-Carlo analysis was performed (see Mueller et al., 2007, for details). To this end, 300 normally distributed flux sets per night were generated, with average and standard deviation matching the measured fluxes and their uncertainties. The NEATM was fitted to each such flux set; best-fit values and statistical uncertainties of $D, p_{V}$, and $\eta$ were taken to be the mean and standard deviation, respectively, of the set of results. See Table 3 for final results.

The accuracy of NEATM-derived diameters and albedos is typically limited by systematic uncertainties, which for observations at phase angles up to $60^{\circ}$ were estimated by Harris (2006) to be $15 \%$ in diameter and $30 \%$ in albedo. The

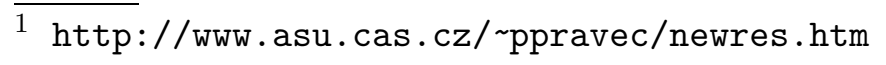


uncertainty in $H(0.5 \mathrm{mag})$ contributes an additional $50 \%$ to the fractional albedo uncertainty but has no significant effect on $D$ or $\eta$ (Harris and Harris, 1997). We adopt as final results the weighted averages of the Sekhmet results presented in Table 3, plus the aforementioned systematics in $D$ and $p_{V}$, leading to $D=1.41 \pm 0.21 \mathrm{~km}$ and $p_{V}=0.23 \pm 0.13$.

(164121) $2003 Y T_{1}$

Thermal-infrared observations of the binary NEA $2003 \mathrm{YT}_{1}$ were obtained on May 8, 2004 using the Thermal Infrared Multi-Mode Instrument (TIMMI2;

Reimann et al., 2000) installed at the ESO 3.6m telescope of the European Southern Observatory (ESO), La Silla, Chile. Photometric observations were carried out in imaging mode through narrow band filters centered at 8.6, 11.9, 12.9, and $17.8 \mu \mathrm{m}$. A standard chopping/nodding observing technique was used similar to that employed in our Sekhmet observations. The Cohen et al. (1995, 1999) flux-calibration standard star $\alpha$ Hya was observed at an airmass very similar to that of the science target.

For data reduction and thermal modeling, we used the same methods and models as in the case of Sekhmet (see previous subsection). Resulting flux values are listed in Table 2 and plotted along the NEATM fit in Figure 1. The resulting diameter, albedo, and $\eta$-value are given in Table 3 along with their statistical uncertainty (from the Monte-Carlo NEATM analysis used for the analysis of the Sekhmet data).

No $H$ value for $2003 \mathrm{YT}_{1}$ is available in the published literature, consequently we used $H=16.2$ as reported by the Minor Planet Center; an uncertainty 
of 0.5 is assumed. Optical lightcurve data, obtained by Galad et al. (2004), indicate a rotation period of 2.343 hours and an amplitude of 0.16 magnitudes. Given that small amplitude, lightcurve-correction of our thermal data was not attempted.

Our final results including the systematic NEATM uncertainty (see above) and the $H$-induced albedo uncertainty are $D=1.56 \pm 0.31 \mathrm{~km}, p_{V}=0.24 \pm 0.16$, and $\eta=2.06 \pm 0.51$

(153591) $2001 S N_{263}$

This triple NEA (Nolan et al., 2008) was observed on 27 Feb 2008 at 7:34 UT, by Neil Dello Russo and Ronald Vervack using the IRTF. They used the cross-dispersed (LXD) mode of SpeX (Rayner et al., 2003) covering 1.94.1 $\mu \mathrm{m}$. Observations are made at two positions along the slit, 15 seconds each, and subtracted pair-wise to correct for sky background. The data were reduced using Spextool (Cushing et al., 2004). Eight pairs of asteroid images were averaged, and the spectra extracted for each of the 5 spectral orders. Following the method of Volquardsen et al. (2004), we fitted the telluric lines separately for the asteroid and standard star spectra, in order to remove them, and combined the orders into a single spectrum. The star spectrum was shifted to match the asteroid spectrum in wavelength. The asteroid spectrum was divided by the star spectrum, resulting in relative reflectance. The spectrum was normalized to 1.0 at 2.4 microns.

Our observations of $2001 \mathrm{SN}_{263}$ are spectroscopic without absolute flux calibration, and therefore do not contain direct information on asteroid diameter. 
In the usable wavelength range $(\sim 2.3-4 \mu \mathrm{m})$, both reflected sunlight and thermal emission contribute to asteroid flux. Reflected and thermal flux dominate at short ( $K$ band) and long ( $L$ band) wavelengths, respectively. We can therefore extract the $K$-band albedo and the NEATM $\eta$ parameter following similar work by, e.g., Howell et al. (1994); Rivkin et al. (2005); Fernández et al. (2006); Volquardsen et al. (2007).

It is worth mentioning that while we measure the $K$-band geometric albedo $p_{K}$, the thermal model needs its $V$-band counterpart $p_{V}$ as an input parameter. Reddy et al. (2008) report an essentially featureless (C-type like) spectrum between $\sim 0.7$ and $\sim 2.2 \mu \mathrm{m}$ with a blue slope of $-0.029 \% / \mu \mathrm{m}$ (Reddy et al., 2008) ${ }^{2}$, implying $p_{R} / p_{K} \sim 1.047$. Hicks et al. (personal communication, 2009) determined $V-R=0.353 \pm 0.015$, implying $p_{V} / p_{R} \sim 1.013$ assuming a solar $V-R$ of 0.367 . We therefore assume $p_{V} / p_{K}=1.061$.

We assumed the reflected component to be spectrally flat across the observed wavelength range. From spectroscopy of main-belt $\mathrm{C}$ types (Rivkin et al., 2002, 2006), we estimate that our assumption is reasonable for the wavelength range shortward of $\sim 2.6 \mu \mathrm{m}$, which is dominated by reflected solar flux. See below for longer wavelengths. Thermal fluxes were calculated using the NEATM. Total fluxes were divided by the solar spectrum as compiled recently by Rieke et al. (2008). Over a grid of $p_{V}$ and $\eta$ values we calculated $\chi^{2}$ given the measured spectrum and its uncertainties, where we allowed our model values to be rescaled by an arbitrary (spectrally constant) factor.

$\overline{2}$ The slope given in Reddy et al. (2008) reads -0.29; Reddy (personal communication, 2009) informed us that it contains a typo (one zero is missing) and that the units are as given in this manuscript. 
The best-fit parameters with formal $1 \sigma$ uncertainties (parameter range for which $\left.\chi^{2} \leq 1+\min \left(\chi^{2}\right)\right)$ are $p_{V}=0.0483 \pm 0.0026$ and $\eta=1.348 \pm 0.030$. We caution that our assumption of a flat reflectance spectrum would be expected to induce additional uncertainty. In order to study its effect, we repeated our analysis excluding the wavelength range 2.8 $3.2 \mu \mathrm{m}$ around the common $3 \mu \mathrm{m}$ feature. Results from that run are indistinguishable from our previous results. Any possible reflectance features at longer wavelengths should not matter given the vast dominance of thermal flux over reflected flux.

Including the systematic albedo uncertainty of $30 \%$ (see above), we obtain $p_{V}=0.048 \pm 0.015$, perfectly consistent with the estimate by Reddy et al. (2008), who obtain $0.05 \pm 0.01$, and with a C-type classification.

Using $H=16.83 \pm 0.04$, (M. Hicks, personal communication, based on observations on 2007 Dec 16 UT) this implies an area-equivalent diameter of the triple system of $2.63 \pm 0.40 \mathrm{~km}$.

Radar observations (Nolan et al., 2008) show the three diameters to be around 2,1 , and $0.4 \mathrm{~km}$, respectively, or an area-equivalent system diameter of $\sqrt{2^{2}+1^{2}+0.4^{2}} \mathrm{~km}=2.3 \mathrm{~km}$, consistent with our result.

\section{Determination of the thermal inertia of binary NEAs}

The distribution of $\eta$-values as a function of $\alpha$ is shown in Fig. 2 ; known binary systems are denoted by solid symbols, all other objects are denoted by open symbols. Where available, uncertainties in $\eta$ are taken from the original works, otherwise we assume a conservative uncertainty of $20 \%$. Note that bi- 
nary NEAs tend to have in general higher $\eta$-values than non-binary NEAs in the same range of $\alpha$. This indicates that binary NEAs present lower color temperatures to the observer than non-binary NEAs. This effect can be explained in terms of above-average thermal inertia and/or a more rapid rotation rate. In order to determine if the higher-than-average $\eta$-values of binary NEAs is not an effect of rapid rotation we plotted $\eta$-values of binary and non-binary NEAs as function of the asteroid rotation rate (when rotation rates were known) in Fig 3. In the range of rotation periods shorter than 3-4 hours, binaries have, in general, $\eta$-values larger than those of non-binary NEAs. Figure 3 also shows a group of slow rotator NEAs $(P>10$ hours) with large $\eta$-yalues, suggesting a high thermal inertia for these objects (Delbo' et al., 2003, 2007). This group of objects includes (2100) Ra-Shalom for which several studies indicate a thermal inertia higher than $\sim 600 \mathrm{Jm}^{-2} s^{-0.5} K^{-1}$ (Shepard et al., 2008; Lim et al., 2005; Harris et al., 1998). In Fig. 4 we plot NEA $\eta$ values against the size of the objects. Note that binary NEAs in the size range between $\sim 1$ and $\sim 5$ $\mathrm{km}$ have in general $\eta$-values higher than those of non-binary, non-slow-rotator NEAs. This suggests that the higher $\eta$-values of binary NEAs is not due to a size effect, as it is known that the thermal inertia of asteroids is size dependent (Delbo' et al., 2007).

We applied a two-dimensional Kolmogorov-Smirnov test (K-S; see Press et al. 1992) on the sample of binaries and slow rotators $(\mathrm{T}>10 \mathrm{~h})$ grouped together against the sample of all other NEOs for which we have $\eta$-values. The significance level for the data in Fig. (2) is about 0.002. This establishes to a near-certainty that the binaries and slow rotators grouped together and the other NEAs were drawn from different populations. If the K-S test is applied to the group of binary NEAs (taken by itself) against the 
sample of non-binary and non-slow-rotator NEAs the hypothesis that the two groups of objects are drawn from the same population can be rejected at $75 \%$ of significance level.

In order to derive the value of the thermal inertia of our sample of binary NEAs, we apply the statistical inversion method developed by Delbo' et al. (2007). The procedure requires $\eta$ to be computed for all members of a synthetic population of asteroids as function of $\Gamma$. The calculation of $\eta$ is performed by numerically generating thermal-infrared spectra by means of a TPM and fitting them with the NEATM. In general $\eta$ depends on $\bar{\theta}, P, A$, the thermalinfrared emissivity, $\epsilon$, the heliocentric distance, $r$, the gross shape of the body, $\Sigma$, the sub-solar latitude, $\theta_{\mathrm{SS}}$, the longitude, $\phi_{\mathrm{SE}}$, and the latitude, $\theta_{\mathrm{SE}}$, of the sub-Earth point (Delbo' et al., 2007). Note that $\theta_{\mathrm{SS}}, \phi_{\mathrm{SE}}$, and $\theta_{\mathrm{SE}}$, depend on the ecliptic longitude $\lambda_{0}$ and latitude $\beta_{0}$ of the pole of the body. As discussed by Delbo' et al. (2007), the parameters $A, \epsilon, r, \bar{\theta}$, and $\Sigma$ contribute little to the variation of $\eta$. Therefore, they have been kept fixed to their nominal values, namely $A=0.073, r=1.2 \mathrm{AU}, \bar{\theta}=36^{\circ}$ and $\Sigma=$ sphere.

For each value of $\Gamma$, we generated a large number $(30,000)$ of synthetic objects whose $P, \theta_{\mathrm{SS}}, \phi_{\mathrm{SE}}$, and $\theta_{\mathrm{SE}}$ parameters have random values with distributions that have been chosen to provide a reasonable match to the population of binary NEAs included in this work. In particular, the distributions of the angles $\theta_{\mathrm{SS}}, \phi_{\mathrm{SE}}$, and $\theta_{\mathrm{SE}}$ were computed starting from the distribution of the spin-axis orientation of NEAs $\left(\lambda_{0}, \beta_{0}\right)$ from La Spina et al. (2004), the phase angle, the heliocentric ecliptic latitude, and the geocentric ecliptic latitude of the asteroids at the time of the infrared observations; the distribution of $P$ was calculated starting from the distribution of the binary NEA rotation periods included in this work (see Table 3). 
The distance $D$ of the two-dimensional Kolmogorov-Smirnov metric (see Press et al. 1992) is used as goodness-of-fit estimator to compare the observed data with the distribution of synthetic points in the $(\eta, \alpha)$ plane. In particular, the fitting procedure consisted in finding the value of $\Gamma$ that minimizes the K-S distance $D$. Figure 5 , where we have plotted the K-S distance $D$ as a function of $\Gamma$, shows that the function $D(\Gamma)$ (continuous line for the nominal spin vector distribution) has a minimum at $\Gamma \sim 480 \mathrm{Jm}^{-2} \mathrm{~s}^{-0.5} \mathrm{~K}^{-1}$, our best estimate of the thermal inertia of binary NEAs. Note that this is more than twice as large as the best-fit thermal inertia of all $\operatorname{NEAs}\left(\Gamma=200 \pm 40 J m^{-2} s^{-0.5} K^{-1}\right)$ for which we have $\eta$ values (dotted line of Fig. 5, taken from Delbo' et al. (2007).

As discussed above, this difference in thermal inertia is neither an artifact of object size nor of spin period. We do not have information about the spin vector of the binary NEAs studied in this work. However, if most of these objects are in, or have been in a YORP-state, there is reason to assume that the direction of their spin vectors are polarized perpendicular to their orbital plane (implying $\theta_{\mathrm{SS}}=0^{\circ}$ ), which is an end state of the YORP effect (Čapek and Vokrouhlický, 2004). For this reason we investigated the sensitivity of our best-fit value of thermal inertia to changes in the input distributions of binary NEAs spin-axis orientations. The dashed line of Fig. 5 shows the function $D(\Gamma)$ obtained by using $\theta_{\mathrm{SS}}=0^{\circ}$ for all synthetic asteroids. In that case, the best-fit thermal inertia decreases to $\sim 400 \mathrm{Jm}^{-2} \mathrm{~s}^{-0.5} \mathrm{~K}^{-1}$, which can be considered as a lower limit for the value of $\Gamma$ for our binary NEAs.

The sensitivity of the best-fit value of $\Gamma$ to the errors affecting the $\eta$-values of Fig. (2) was studied by performing a Monte Carlo simulations, in which we randomly varied the values of the $\eta$-values within their error bars (assuming normally distributed random numbers), 
and for each simulation of noise-corrupted data we calculated the best-fit thermal inertia. The standard deviation of $\Gamma$ was found to be $70 \mathrm{Jm}^{-2} \mathrm{~s}^{-0.5} \mathrm{~K}^{-1}$.

\section{Discussion}

We showed that our sample of binary NEAs has a higher thermal inertia than their non-binary counterparts. The difference is statistically significant as evidenced by a K-S test; it is not an artifact of object size, spin rate, or observing conditions. Why would binary NEAs have an elevated thermal inertia?

In the context of the YORP binary formation model of Walsh et al. (2008), this can be explained by much of the main body's surface regolith being removed during the binary formation process. The final destination of the regolith is an interesting question. If the regolith does move and is ejected similar to the boulders in the Walsh et al. (2008) model, then it is being ejected off the primary continuously during satellite formation. Once the seed for the satellite forms, it will be quite effective in accreting this material. The time scale for bulding up the satellite is of the order of the YORP spin-up time scale $\left(10^{4}-10^{6}\right.$ years Walsh et al. 2008).

So it is possible that most of the primary's removed regolith stays in the binary system, but is sequestered inside the secondary. Observations of the system are dominated by the surface of the primary which has largely lost its regolith, so the effects of depletion on the primary are captured in the thermal observations. 
If the regolith was removed during spin-up, before the binary formation begins, then other rapidly rotating NEAs would have surface properties similar to those of the binaries. This is not seen in the data, as there is a strong distinction between binary NEAs and rapidly rotating NEAs. Similarly, if regolith was not removed during the spin-up or binary formation, then there should be no noticeable thermal differences between a sample of binaries and non-binary NEAs. Again, this is not seen, as NEAs with moderate spin rates have normal $\eta$ values.

Apollo

Apollo has the lowest $\eta$ value in our sample of binaries, and one which is not significantly higher than the group of rapidly rotating NEAs. In comparison to the other systems, and NEA binaries in general, the Apollo system has a very small secondary, estimated to be only $5 \%$ the size of the primary, and therefore only $\sim 0.0125 \%$ the mass of the primary (Ostro et al., 2005). So the low $\eta$ value for Apollo could be a sign that it is just at the beginning of its binary formation cycle and has not yet lost much regolith. It has been observed to have a YORP-induced increasing spin rate, so it may still be spinning up and just starting to transfer mass to a newly formed secondary (Kaasalainen et al., 2007).

$2001 S N_{263}$

$2001 \mathrm{SN}_{263}$ is the only known triple NEA. The outermost satellite is quite distant with a semi-major axis $\sim 12-14$ primary radii, while the inner, smaller, 
satellite orbits at only 3-4 primary radii (Nolan et al., 2008). This appears to be a highly evolved YORP formed case, where a satellite was formed, and then evolved far enough away from the primary to allow a second satellite to form. Therefore this unique system could potentially be the one most dramatically affected by the regolith depletion mechanism outlined in this work. Its $\eta$ value is $1.35 \pm 0.03$, and not entirely suggestive of an extreme case of regolith depletion.

Slow rotators

The other group of NEAs with high measured $\eta$ values are slow rotators. The binary systems were exceptional when compared to other NEAs of similar spin rates, but the group of slow rotators have similarly high $\eta$ values. This group of NEAs are interesting as the YORP-effect has successfully been used to explain the excess of slow-rotating asteroids among NEAs (Pravec et al., 2008). The details of what happens to the asteroid as YORP pushes it to very slow spin rates is still somewhat unclear. The YORP-induced spin-rate change is practically independent of thermal inertia, but the YORP-induced obliquity change is not (Čapek and Vokrouhlický, 2004). However, with the data presented in this work, all of the bodies with high $\eta$, and likely high thermal inertia, are bodies which probably have had their spin rates directly altered by YORP. Certainly, with the small number of observations presented here, this is not evidence that thermal inertia plays a role in the effectiveness of YORP, but simply an observation that all the bodies in our sample with high $\eta$ and high thermal inertia appear to be the ones most strongly suspected of being in a YORP spin-up or spin-down cycle. 


\section{Conclusions}

We show that the typical thermal inertia of our sample of binary NEAs is around $480 \pm 70 \mathrm{Jm}^{-2} \mathrm{~s}^{-0.5} \mathrm{~K}^{-1}$ (one sigma), or more than twice the value found to be typical for NEAs in general. Compared to non-binary NEAs, binaries display a relative lack of fine regolith indicative of regolith movement. It is reasonable to assume that this is associated with the binary formation process. Indeed, numerical modeling of binary formation through YORP-induced disruption suggests that such surface alterations occur before and during binary formation. Our results therefore provide support for these models.

Moreover, we suggest that every high- $\eta$ fast-rotating NEA is very likely to be binary, making it a prime target for attempting a detection of a satellite through photometric lightcurves or radar.

Lastly this work produced some new questions for investigation, primarily about the interesting elevated $\eta$-value of NEAs with rotation periods longer

than $10 \mathrm{~h}$. Though these bodies do not directly fall into the YORP-spinup category like the binaries, they are an interesting population of bodies which are possibly tied to a YORP-cycle affecting the spin rates of a larger percentage of NEAs. However, more data are needed with this population as we only present three measurements.

\section{Acknowledgments}

This research was carried out while Marco Delbo', Kevin Walsh and Michael Mueller were Henri Poincaré Fellows at the Observatoire de la Côte d'Azur. 
The Henri Poincaré Fellowship is funded by the CNRS-INSU, the Conseil Général des Alpes-Maritimes and the Rotary International - District 1730.

M.D.B. wishes to thank G.P. Tozzi (PI of the ESO program) for the observation of the asteroid $2003 \mathrm{YT}_{1}$. We thank N. Russo and R. Vervack for kindly providing their SpeX data of $2001 \mathrm{SN}_{263}$.

We also wish to thank Lucy Lim and an anonymous referee for suggestions that led to significant improvements in the paper. 


\section{References}

Abe, M., Sato, I., Araki, H., 2000. Lightcurve and Color of Near-Earth-Asteroid 1989ML. Adv. Space Res. 25, 269-272.

Becker, T., Howell, E. S., Nolan, M. C., Magri, C., 2008. Physical Modeling of Triple Near-Earth Asteroid 153591 (2001 SN263). Bull. Am. Astron. Soc. 40, 437.

Benner, L. A. M., Nolan, M. C., Ostro, S. J., Giorgini, J. D., Margot, J. L., 2001. 1998 ST_27. IAU Circ. 7730, 2.

Burns, J. A., Lamy, P. L., Soter, S., 1979. Radiation forces on small particles in the solar system. Icarus 40, 1-48.

Čapek, D., Vokrouhlický, D., 2004. The YORP effect with finite thermal conductivity. Icarus $172,526-536$.

Christensen, P. R., and 21 colleagues, 2003. Morphology and Composition of the Surface of Mars: Mars Odyssey THEMIS Results. Science 300, 2056-2061.

Cohen, M., Walker, R. G., Carter, B., Hammersley, P., Kidger, M., Noguchi, K., 1999. Spectral Irradiance Calibration in the Infrared. X. A Self-Consistent Radiometric All-Sky Network of Absolutely Calibrated Stellar Spectra. Astron. J. 117, 1864-1889.

Cohen, M., Witteborn, F. C., Walker, R. G., Bregman, J. D., Wooden, D. H., 1995. Spectral Irradiance Calibration in the Infrared.IV. 1.2-35 micron spectra of six standard stars. Astron. J. 110, 275-289.

Cushing, M. C., Vacca, W. D., Rayner, J. T., 2004. Spextool: A spectral extraction package for spex, a 0.8-5.5 micron cross-dispersed spectrograph. Publ. Astron. Soc. Pacific 116, 362-376.

Delbo', M., 2004. The nature of near-earth asteroids from the study of their thermal infrared emission. Ph.D. Thesis.

Delbo', M., Dell'Oro, A., Harris, A. W., Mottola, S., Mueller, M., 2007. Thermal inertia of near-Earth asteroids and implications for the magnitude of the Yarkovsky effect. Icarus 190, 236-249.

Delbo', M., Harris, A. W., 2002. Physical properties of near-Earth asteroids from thermal infrared observations and thermal modeling. Meteoritics and Planetary Science 37, 1929-1936.

Delbo', M., Harris, A. W., Binzel, R. P., Pravec, P., Davies, J. K., xs2003. Keck observations of near-Earth asteroids in the thermal infrared. Icarus 166, 116-130.

Delbo', M., Tanga, 2009. Thermal inertia of main belt asteroids smaller than 100 $\mathrm{km}$ from IRAS data. Planet. Space Sci. 57, 259-265

Emery, J. P., Sprague, A. L., Witteborn, F. C., Colwell, J. E., Kozlowski, R. W. H., Wooden, D. H., 1998. Mercury: Thermal Modeling and Mid-infrared (5-12 $\mu \mathrm{m})$ Observations. Icarus 136, 104-123.

Fernández, Y. R., Campins, H., Kassis, M., Hergenrother, C. W., Binzel, R. P., Licandro, J., Hora, J. L., Adams, J. D., 2006. Comet 162P/Siding Spring: A Surprisingly Large Nucleus. Astron. J. 132, 1354-1360.

Galad, A., Gajdos, S., Kornos, L., Vilagi, J., Pravec, P., 2004. 2003 YT_1. IAU Circ. 8336, 4.

Harris, A. W., 1998. A Thermal Model for Near-Earth Asteroids. Icarus 131, 291301.

Harris, A. W., 2006. The surface properties of small asteroids from thermal-infrared observations. in Proc. of IAU Symposium 229, eds. D. Lazzaro, et al. (Cambridge: Cambridge University Press), 449-463.

Harris, A. W., Davies, J. K., 1999. Physical Characteristics of Near-Earth Asteroids 
from Thermal Infrared Spectrophotometry. Icarus 142, 464-475.

Harris, A. W., Davies, J. K., Green, S. F., 1998. Thermal Infrared Spectrophotometry of the Near-Earth Asteroids 2100 Ra-Shalom and 1991 EE. Icarus 135, 441-450.

Harris, A. W., Harris, A. W., 1997. On the Revision of Radiometric Albedos and Diameters of Asteroids. Icarus 126, 450-454.

Harris, A. W., Young, J. W., Goguen, J., Hammel, H. B., Hahn, G., Tedesco, E. F., Tholen, D. J., 1987. Photoelectric lightcurves of the asteroid 1862 Apollo. Icarus 70, 246-256.

Howell, E. S., Britt, D. T., Bell, J. F., Binzel, R. P., Lebofsky, L. A., 1994. Visible and near-infrared spectral observations of 4179 Toutatis. Icarus 111, 468-474.

Jakosky, B. M., 1986. On the thermal properties of Martian fines. Icarus 66, 117124.

Kaasalainen, M., Duurech, J., Warner, B. D., Krugly, Y. N., Gaftonyuk, N. M., 2007. Acceleration of the rotation of asteroid 1862 Apollo by radiation torques. Nature 446, 420-422.

Krisciunas, K., Sinton, W., Tholen, K., Tokunaga, A., Golisch, W., Griep, D., Kaminski, C., Impey, C., Christian, C., 1987. Atmospheric Extinction and NightSky Brightness at Mauna-Kea. Publ. Astron. Soc. Pacific 99, 887-894.

Kührt, E., Giese, B., 1989. A thermal model of the Martian satellites. Icarus 81, 102-112.

La Spina, A., Paolicchi, P., Kryszczyńska, A., Pravec, P., 2004. Retrograde spins of near-Earth asteroids from the Yarkovsky effect. Nature 428, 400-401.

Lagerros, J. S. V., 1996. Thermal physics of asteroids. I. Effects of shape, heat conduction and beaming. Astron. Astrophys. 310, 1011-1020.

Lim, L. F., Emery, J. P., McConnochie, T. H., 2005. Thermal Infrared (8-13 micron) Spectra of the NEA 2100 Ra-Shalom. Bull. Am. Astron. Soc. 37, p. 629.

Lowry, S. C., and 10 colleagues, 2007. Direct Detection of the Asteroidal YORP Effect. Science 316, 272-274.

Mellon, M. T., Jakosky, B. M., Kieffer, H. H., Christensen, P. R., 2000. HighResolution Thermal Inertia Mapping from the Mars Global Surveyor Thermal Emission Spectrometer. Icarus 148, 437-455.

Mueller, M., 2007. Surface Properties of Asteroids from Mid-Infrared Observations and Thermophysical Modeling. Ph.D. Thesis.

Mueller, M., Harris, A. W., Fitzsimmons, A., 2007. Size, albedo, and taxonomic type of potential spacecraft target Asteroid (10302) 1989 ML. Icarus 187, 611-615.

Neish, C. D., Nolan, M. C., Howell, E. S., Rivkin, A. S., 2003. Radar Observations of Binary Asteroid 5381 Sekhmet. Bull. Am. Astron. Soc. 35 pp. 1421.

Nolan, M. C., Howell, E. S., Becker, T. M., Magri, C., Giorgini, J. D., Margot, J. L., 2008. Arecibo Radar Observations of $2001 \mathrm{SN}_{263}$ : A Near-Earth Triple Asteroid System. In: AAS/Division for Planetary Sciences Meeting Abstracts. Vol. 40 \#25.04.

Nolan, M. C., Howell, E. S., Magri, C., Beeney, B., Campbell, D. B., Benner, L. A. M., Ostro, S. J., Giorgini, J. D., Margot, J.-L., 2002. 2002 BM 26. IAU Circ. xs7824, 1.

Noll, K. S., 2006. Solar System binaries. in Proc. of IAU Symposium 229, eds. D. Lazzaro, et al. (Cambridge: Cambridge University Press), 301-318.

Ostro, S. J., Benner, L. A. M., Giorgini, J. D., Nolan, M. C., Hine, A. A., Howell, E. S., Margot, J. L., Magri, C., Shepard, M. K., 2005. (1862) Apollo. IAU Circ. 8627, 2. 
Pravec, P., Harris, A. W., 2007. Binary asteroid population. 1. Angular momentum content. Icarus 190, 250-259.

Pravec, P., Harris, A. W., Michalowski, T., 2002. Asteroid Rotations. In: Bottke Jr., W .F., Cellino, A., Paolicchi, P., Binzel, R. P. (Eds.), Asteroids III. Univ. of Arizona Press, Tucson. pp. 113-122.

Pravec, P., and 30 colleagues, 2008. Spin rate distribution of small asteroids. Icarus 197, 497-504.

Pravec, P., Harris, A. W., Warner, B. D., 2007. NEA rotations and binaries. In: Valsecchi, G. B., Vokrouhlický, D. (Eds.), IAU Symposium. 236, 167-176.

Pravec, P., and 56 colleagues, 2006. Photometric survey of binary near-Earth asteroids. Icarus 181, 63-93.

Pravec, P., Wolf, M., Sarounova, L., 1998. Lightcurves of 26 Near-Earth Asteroids. Icarus 136, 124-153.

Presley, M. A., Christensen, P. R., 1997a. Thermal conductivity measurements of particulate materials 1. A review. JGR 102, 6535-6550.

Presley, M. A., Christensen, P. R., 1997b. Thermal conductivity measurements of particulate materials 2 . results. JGR 102, 6551-6566.

Press, W. H., Teukolsky, S. A., Vetterling, W. T., Flannery, B. P., 1992. Numerical recipes in FORTRAN. The art of scientific computing. Cambridge: University Press, 1992, 2nd ed.

Rayner, J. T., Toomey, D. W., Onaka, P. M., Denault, A. J., Stahlberger, W. E., Vacca, W. D., Cushing, M. C., Wang, S., 2003. Spex: A medium-resolution 0.85.5 micron spectrograph and imager for the nasa infrared telescope facility. Publ. Astron. Soc. Pacific 115, 362-382.

Reddy, V., Gaffey, M. J., Schaal, M., Takir, D., 2008. Physical Characterization of First Triplet Near-Earth Asteroid (153591) $2001 \mathrm{SN}_{263}$. LPI Contributions 1405, 8244.

Reimann, H.-G., Linz, H., Wagner, R., Relke, H., Kaeufl, H. U., Dietzsch, E., Sperl, M., Hron, J., 2000. TIMMI2: a new multimode mid-infrared instrument for the ESO 3.6-m telescope. In: Iye, M., Moorwood, A. F. (Eds.), Society of PhotoOptical Instrumentation Engineers (SPIE) Conference Series. Vol. 4008 of Society of Photo-Optical Instrumentation Engineers (SPIE) Conference Series. pp. 11321143.

Ressler, M. E., Werner, M. W., Van Cleve, J., Chou, H. A., Mar. 1994. The JPL deep-well mid-infrared array camera. Experimental Astronomy 3, 277-280.

Richardson, D. C., Walsh, K. J., 2006. Binary Minor Planets. Annual Review of Earth and Planetary Sciences 34, 47-81.

Rieke, G. H. and 13 colleagues, 2008. Absolute Physical Calibration in the Infrared. Astron. J. 135, 2245-2263.

Rivkin, A. S., Binzel, R. P., Bus, S. J., 2005. Constraining near-Earth object albedos using near-infrared spectroscopy. Icarus 175, 175-180.

Rivkin, A. S., Howell, E. S., Vilas, F., Lebofsky, L. A., 2002. Hydrated Minerals on Asteroids: The Astronomical Record. Asteroids III, 235-253.

Rivkin, A. S., Volquardsen, E. L., Clark, B. E., 2006. The surface composition of Ceres: Discovery of carbonates and iron-rich clays. Icarus 185, 563-567.

Rubincam, D. P., 2000. Radiative Spin-up and Spin-down of Small Asteroids. Icarus 148, 2-11.

Rubincam, D. P., Rowlands, D. D., Ray, R. D., 2002. Is asteroid 951 Gaspra in a resonant obliquity state with its spin increasing due to YORP? Journal of Geophysical Research (Planets) 107, 5065. 
Scheeres, D. J., 2007. Rotational fission of contact binary asteroids. Icarus 189, 370-385.

Shepard, M. K., Clark, B. E., Nolan, M. C., Benner, L. A. M., Ostro, S. J., Giorgini, J. D., Vilas, F., Jarvis, K., Lederer, S., Lim, L. F., McConnochie, T., Bell, J., Margot, J.-L., Rivkin, A., Magri, C., Scheeres, D., Pravec, P., 2008. Multi-wavelength observations of Asteroid 2100 Ra-Shalom. Icarus 193, 20-38.

Spencer, J. R., 1990. A rough-surface thermophysical model for airless planets. Icarus 83, 27-38.

Spencer, J. R., Lebofsky, L. A., Sykes, M. V., 1989. Systematic biases in radiometric diameter determinations. Icarus 78, 337-354.

Statler, T. S., Aug. 2009. Extreme sensitivity of the YORP effect to small-scale topography. Icarus 202, 502-513.

Szabó, G. M., Csák, B., Sárneczky, K., Kiss, L. L., Aug. 2001. Photometric observations of 9 Near-Earth Objects. Astron. Astrophys. 375, 285-292.

Taylor, P. A., and 11 colleagues, 2007. Spin Rate of Asteroid (54509) 2000 PH5 Increasing Due to the YORP Effect. Science 316, 274-277.

Veverka and 32 colleagues, 2001a. Imaging of Small-Scale Features on 433 Eros from NEAR: Evidence for a Complex Regolith. Science 292, 484-488.

Veverka and 38 colleagues, 2001b. The landing of the NEAR-Shoemaker spacecraft on asteroid 433 Eros. Nature 413, 390-393.

Volquardsen, E. L., Rivkin, A. S., Bus, S. J., 2004. Improved 3 micron Band Determination in the Spectra of Aqueously Altered Asteroid Regoliths. In: Bull. Am. Astron. Soc. 36, 1140.

Volquardsen, E. L., Rivkin, A. S., Bus, S. J., 2007. Composition of hydrated nearEarth object (100085) 1992 UY4. Icarus 187, 464-468.

Walsh, K. J., Richardson, D. C., Michel, P., 2008. Rotational breakup as the origin of small binary asteroids. Nature 454, 188-191.

Weissman, P., Doressoundiram, A., Hicks, M., Chamberlin, A., Sykes, M., Larson, S., Hergenrother, C., Sep. 1999. CCD Photometry of Comet and Asteroid Targets of Spacecraft Missions. In: AAS/Division for Planetary Sciences Meeting Abstracts. Vol. 31 \#30.03.

Winter, D. F., Krupp, J. A., 1971. Directional characteristics of infrared emission from the moon. Moon 2, 279-292.

Wolters, S. D., Green, S. F., McBride, N., Davies, J. K., 2005. Optical and thermal infrared observations of six near-Earth asteroids in 2002. Icarus 175, 92-110.

Yano and 19 collegues, 2006. Touchdown of the Hayabusa Spacecraft at the Muses Sea on Itokawa. Science 312, 1350-1353. 


\section{Tables}

Table 1

\begin{tabular}{|r|r|rrr|}
\hline Object & Time (UT) & $r(\mathrm{AU})$ & $\Delta(\mathrm{AU})$ & $\alpha\left(^{\circ}\right)$ \\
\hline (5381) Sekhmet & 2003 May 12 14h & 1.1138 & 0.1459 & 42.0 \\
& 2003 May 13 14h & 1.1174 & 0.1400 & 37.8 \\
& 2003 May 14 13h & 1.1207 & 0.1354 & 33.5 \\
& 2003 May 15 14h & 1.1243 & 0.1316 & 28.7 \\
& 2003 May 16 11h & 1.1273 & 0.1296 & 24.7 \\
\hline (164121) 2003 YT 1 & 2004 May 08 02h & 1.0359 & 0.1242 & 74.3 \\
\hline (153591)2001 $\mathrm{SN}_{263}$ & 2008 Feb 27 07h & 1.0520 & 0.0705 & 27.4 \\
\hline
\end{tabular}

Observational circumstances for the thermal-IR observations reported here-in: heliocentric distance $r$, geocentric distance $\Delta$, and solar phase angle $\alpha$. 


\begin{tabular}{|c|c|c|c|c|c|}
\hline Object & Date & Time (UT) & Wavelength $(\mu m)$ & Flux (Jy) & Error $(\mathrm{Jy})$ \\
\hline \multirow[t]{39}{*}{ (5381) Sekhmet } & 2003-05-12 & $13: 53: 36$ & 11.70 & 0.927 & 0.017 \\
\hline & 2003-05-12 & 14:14:05 & 8.81 & 0.548 & 0.012 \\
\hline & 2003-05-12 & $14: 25: 01$ & 11.70 & 0.903 & 0.026 \\
\hline & 2003-05-12 & $14: 34: 54$ & 10.27 & 0.748 & 0.025 \\
\hline & 2003-05-12 & $14: 43: 51$ & 10.27 & 0.795 & 0.026 \\
\hline & 2003-05-12 & $14: 54: 24$ & 11.70 & 0.888 & 0.017 \\
\hline & 2003-05-12 & $15: 06: 44$ & 8.81 & 0.496 & 0.015 \\
\hline & 2003-05-12 & $15: 16: 11$ & 10.27 & 0.763 & 0.025 \\
\hline & 2003-05-13 & $13: 24: 17$ & 11.70 & 0.980 & 0.018 \\
\hline & 2003-05-13 & 13:37:00 & 8.81 & 0.588 & 0.016 \\
\hline & 2003-05-13 & $13: 47: 42$ & 11.70 & 0.983 & 0.017 \\
\hline & 2003-05-13 & $13: 57: 16$ & 10.27 & 0.824 & 0.031 \\
\hline & 2003-05-13 & $14: 07: 40$ & 11.70 & 0.935 & 0.019 \\
\hline & 2003-05-13 & $14: 29: 49$ & 11.70 & 0.995 & 0.026 \\
\hline & 2003-05-13 & $14: 42: 30$ & 8.81 & 0.578 & 0.017 \\
\hline & 2003-05-13 & $14: 51: 49$ & 10.27 & 0.884 & 0.038 \\
\hline & 2003-05-13 & $15: 02: 11$ & 11.70 & 1.052 & 0.023 \\
\hline & 2003-05-14 & $10: 56: 58$ & 11.70 & 0.637 & 0.016 \\
\hline & 2003-05-14 & $11: 05: 18$ & 10.27 & 0.647 & 0.036 \\
\hline & 2003-05-14 & $11: 13: 11$ & 10.27 & 0.486 & 0.037 \\
\hline & 2003-05-14 & 11:23:35 & 11.70 & 0.643 & 0.021 \\
\hline & $2003-05-14$ & $11: 34: 14$ & 8.81 & 0.342 & 0.015 \\
\hline & 2003-05-14 & $11: 44: 26$ & 8.81 & 0.383 & 0.018 \\
\hline & 2003-05-14 & 11:55:03 & 11.70 & 0.581 & 0.020 \\
\hline & 2003-05-14 & 12:04:32 & 10.27 & 0.630 & 0.053 \\
\hline & 2003-05-14 & $12: 13: 50$ & 10.27 & 0.518 & 0.044 \\
\hline & 2003-05-14 & $12: 24: 10$ & 11.70 & 0.565 & 0.017 \\
\hline & 2003-05-14 & $12: 35: 16$ & 8.81 & 0.401 & 0.019 \\
\hline & 2003-05-14 & $12: 45: 31$ & 8.81 & 0.363 & 0.018 \\
\hline & 2003-05-14 & $12: 56: 14$ & 11.70 & 0.569 & 0.019 \\
\hline & 2003-05-14 & 13:30:07 & 11.70 & 1.024 & 0.015 \\
\hline & 2003-05-14 & $13: 40: 45$ & 8.81 & 0.711 & 0.015 \\
\hline & 2003-05-14 & $13: 49: 15$ & 10.27 & 1.008 & 0.055 \\
\hline & 2003-05-14 & $14: 10: 52$ & 8.81 & 0.686 & 0.018 \\
\hline & 2003-05-14 & 14:19:19 & 10.27 & 1.099 & 0.055 \\
\hline & 2003-05-14 & $14: 29: 33$ & 11.70 & 1.027 & 0.026 \\
\hline & 2003-05-14 & 14:40:05 & 8.81 & 0.642 & 0.023 \\
\hline & 2003-05-14 & $14: 48: 38$ & 10.27 & 0.941 & 0.037 \\
\hline & 2003-05-14 & $14: 58: 46$ & 11.70 & 1.107 & 0.015 \\
\hline
\end{tabular}




\begin{tabular}{|c|c|c|c|c|c|}
\hline Object & Date & Time (UT) & Wavelength $(\mu m)$ & Flux (Jy) & Error $(\mathrm{Jy})$ \\
\hline & 2003-05-15 & $12: 30: 52$ & 11.70 & 1.253 & 0.017 \\
\hline & 2003-05-15 & $12: 41: 18$ & 8.81 & 0.800 & 0.015 \\
\hline & 2003-05-15 & $12: 49: 52$ & 10.27 & 1.003 & 0.037 \\
\hline & 2003-05-15 & 13:00:14 & 11.70 & 1.221 & 0.017 \\
\hline & 2003-05-15 & $13: 11: 17$ & 8.81 & 0.695 & 0.017 \\
\hline & 2003-05-15 & $13: 19: 51$ & 10.27 & 0.985 & 0.051 \\
\hline & 2003-05-15 & $13: 28: 00$ & 10.27 & 0.978 & 0.047 \\
\hline & 2003-05-15 & $13: 38: 17$ & 11.70 & 1.179 & 0.021 \\
\hline & 2003-05-15 & $13: 51: 41$ & 11.70 & 0.213 & 0.019 \\
\hline & 2003-05-15 & 14:01:55 & 11.70 & 0.215 & 0.019 \\
\hline & 2003-05-15 & $14: 01: 55$ & 11.70 & 0.212 & 0.025 \\
\hline & 2003-05-15 & $14: 12: 37$ & 8.81 & 0.134 & 0.021 \\
\hline & 2003-05-15 & $14: 22: 50$ & 8.81 & 0.153 & 0.019 \\
\hline & 2003-05-15 & $14: 22: 50$ & 8.81 & 0.136 & 0.024 \\
\hline & 2003-05-15 & $14: 31: 29$ & 10.27 & 0.282 & 0.037 \\
\hline & 2003-05-15 & $14: 39: 39$ & 10.27 & 0.153 & 0.023 \\
\hline & 2003-05-15 & 14:39:39 & 10.27 & 0.165 & 0.029 \\
\hline & 2003-05-15 & $14: 49: 59$ & 11.70 & 0.208 & 0.023 \\
\hline & 2003-05-15 & $15: 00: 19$ & 11.70 & 0.150 & 0.020 \\
\hline & 2003-05-15 & $15: 00: 19$ & 11.70 & 0.176 & 0.031 \\
\hline & $2003-05-16$ & $11: 03: 57$ & 11.70 & 1.360 & 0.018 \\
\hline & $2003-05-16$ & $11: 16: 13$ & 8.81 & 0.816 & 0.019 \\
\hline & 2003-05-16 & $11: 24: 58$ & 10.27 & 1.238 & 0.023 \\
\hline \multirow[t]{5}{*}{$(164121) 2003 \mathrm{YT}_{1}$} & 2004-05-08 & $01: 53: 22$ & 11.9 & 0.870 & 0.05 \\
\hline & & 02:00:03 & 8.6 & 0.457 & 0.04 \\
\hline & & 02:06:50 & 12.9 & 0.980 & 0.05 \\
\hline & & $02: 26: 02$ & 17.8 & 1.2 & 0.4 \\
\hline & & 02:31:09 & 11.9 & 0.810 & 0.05 \\
\hline$(153591) 2001 \mathrm{SN}_{263}$ & $2008-02-27$ & $07: 34: 31$ & $2.3-4.0$ & see Fig. 1 & see Fig. 1 \\
\hline
\end{tabular}

Table 2: Observed fluxes. Flux calibration was performed against observations of Cohen et al. (1995, 1999) standard stars $\alpha$ Hya and $\gamma$ Aql for (5381) Sekhmet and of $\alpha$ Hya for (164121) $2003 \mathrm{YT}_{1}$. 


\begin{tabular}{|c|c|c|c|c|c|c|c|}
\hline Object & Night & $\begin{array}{c}\alpha \\
\left({ }^{\circ}\right) \\
\end{array}$ & $\begin{array}{c}D \\
(\mathrm{~km})\end{array}$ & $p_{V}$ & $\eta$ & $\begin{array}{c}P \\
(\mathrm{hrs}) \\
\end{array}$ & Ref. \\
\hline \multicolumn{8}{|l|}{ Binary NEAs } \\
\hline \multirow[t]{5}{*}{ (5381)Sekhmet } & 20030512 & 42 & $1.497 \pm 0.056$ & $0.203 \pm 0.015$ & $1.90 \pm 0.14$ & 2.7053 & $\mathrm{a}, 0$ \\
\hline & 20030513 & 38 & $1.416 \pm 0.056$ & $0.227 \pm 0.018$ & $1.77 \pm 0.14$ & & $y$ \\
\hline & 20030514 & 33 & $1.27 \pm 0.21$ & $0.30 \pm 0.10$ & $1.43 \pm 0.47$ & & $"$ \\
\hline & 20030515 & 29 & $1.355 \pm 0.045$ & $0.247 \pm 0.016$ & $1.58 \pm 0.11$ & & $"$ \\
\hline & 20030516 & 25 & $1.418 \pm 0.062$ & $0.226 \pm 0.020$ & $1.63 \pm 0.14$ & & $"$ \\
\hline $2003 \mathrm{YT}_{1}$ & 20040508 & 74 & $1.561 \pm 0.202$ & $0.240 \pm 0.067$ & $2.06 \pm 0.51$ & 2.343 & $\mathrm{~b}, 0$ \\
\hline $2001 \mathrm{SN}_{263}$ & 20080226 & 27 & $2.63 \pm 0.40$ & $0.048 \pm 0.015$ & $1.35 \pm 0.03$ & 2.60 & $\mathrm{c}, 0$ \\
\hline (1862) Apollo & 19801126 & 35 & 1.4 & 0.26 & $1.15 \pm 0.23$ & 3.0655 & $\mathrm{~d}, 1$ \\
\hline (1866) Sysiphus & 19980629 & 35 & 8.9 & 0.14 & $1.34 \pm 0.2$ & 2.700 & $\mathrm{e}, 2$ \\
\hline (3671) Dionysus & 19970602 & 58 & 1.5 & 0.16 & $3.10 \pm 0.62$ & 2.7053 & $\mathrm{~b}, 3$ \\
\hline $2002 \mathrm{BM}_{26}$ & 20020221 & 60 & 0.84 & 0.023 & $3.10 \pm 0.4$ & 2.7 & $\mathrm{f}, 4$ \\
\hline $1999 \mathrm{HF}_{1}$ & 20020322 & 91 & 4.74 & 0.11 & $1.68 \pm 0.3$ & 2.3192 & $\mathrm{~g}, 5$ \\
\hline \multicolumn{8}{|l|}{ Slow rotators } \\
\hline (86039) $1999 \mathrm{NC}_{43}$ & $17-03-2000$ & 59 & 2.22 & 0.140 & 2.86 & 34.49 & $\mathrm{~h}, 4$ \\
\hline (2100) Ra-Shalom & 21-08-2000 & 39 & 2.79 & 0.080 & 2.32 & 19.8 & $\mathrm{i}, 4$ \\
\hline (2100) Ra-Shalom & 30-08-1997 & 41 & 2.50 & 0.130 & 1.80 & 19.8 & $\mathrm{i}, 1$ \\
\hline $2002 \mathrm{HK}_{12}$ & 28092002 & 33 & 0.80 & 0.170 & 2.84 & 12.69 & h, 5 \\
\hline $1998 \mathrm{ML}$ & $2-3062006$ & 52.3 & $0.28 \pm 0.05$ & $0.37 \pm 0.15$ & $2.5 \pm 0.5$ & 19.0 & $\mathrm{j}, \mathrm{k}, 6$ \\
\hline
\end{tabular}

Table 3: Diameters, albedo, and $\eta$-values from thermal model fit of binary NEAs. The rotation period of each object, the phase angle and the date of the observations are also reported. References: (rotation periods) a) Neish et al. (2003); b) Pravec et al. (2006); c) Nolan et al. (2008); d) Harris et al. (1987); e) Szabó et al. (2001); f) Nolan et al. (2002); g) Pravec et al. (2002); h) Pravec et al. (2005; Pravec, P., Wolf, M., Sarounova, L.: 2005, posted on WWW: http://www.asu.cas.cz/ ppravec/neo.htm); i) Pravec et al. (1998); j) Abe et al. (2000); k) Weissman et al. (1999); (thermal modeling) 0) This work; 1) (Harris, 1998, and references therein); 2) Delbo' (2004); 3) Harris and Davies (1999); 4) Delbo' et al. (2003); 5) Wolters et al. (2005); 6) Mueller et al. (2007). 


\section{Figures}

Sekhmet at MIRLIN on May 12, 2003

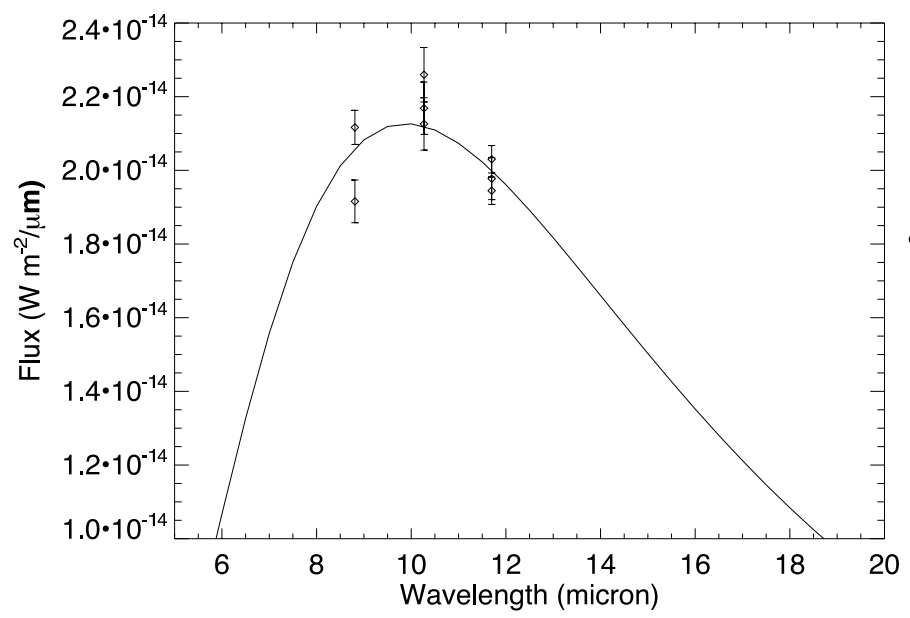

$2003 \mathrm{YT}_{1}$ at TIMMI2 on May 8, 2004

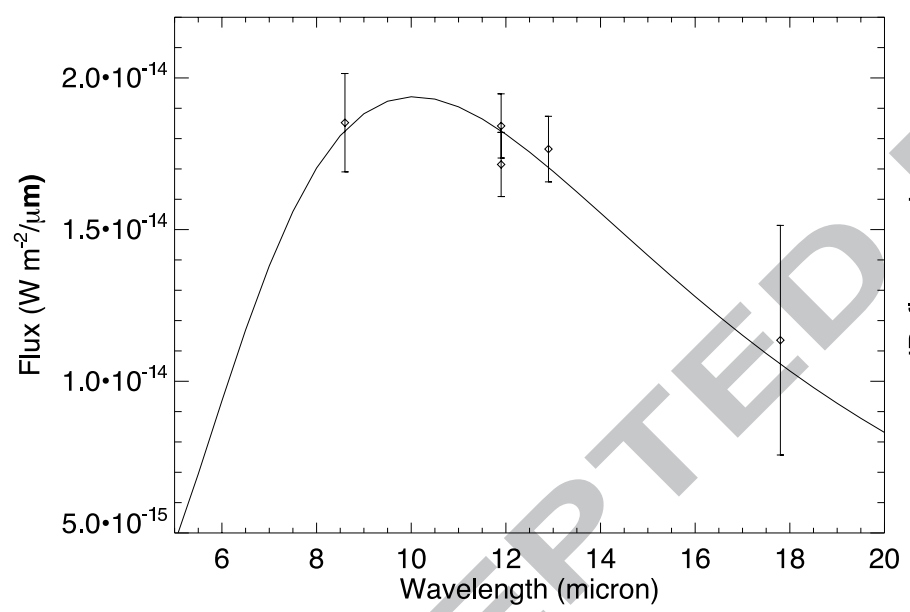

Sekhmet at MIRLIN on May 16, 2003

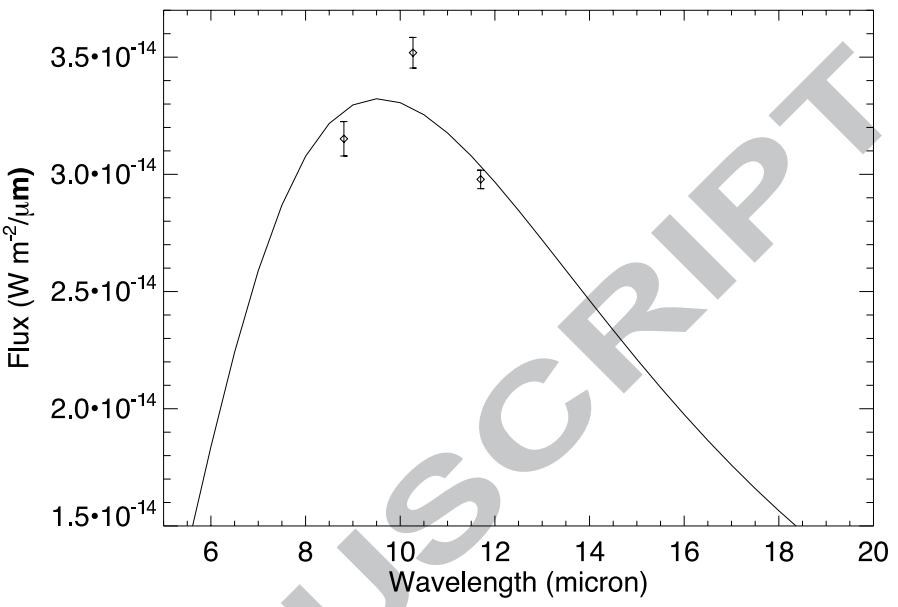

$2001 \mathrm{SN}_{263}$ at SpeX on Feb 27, 2008

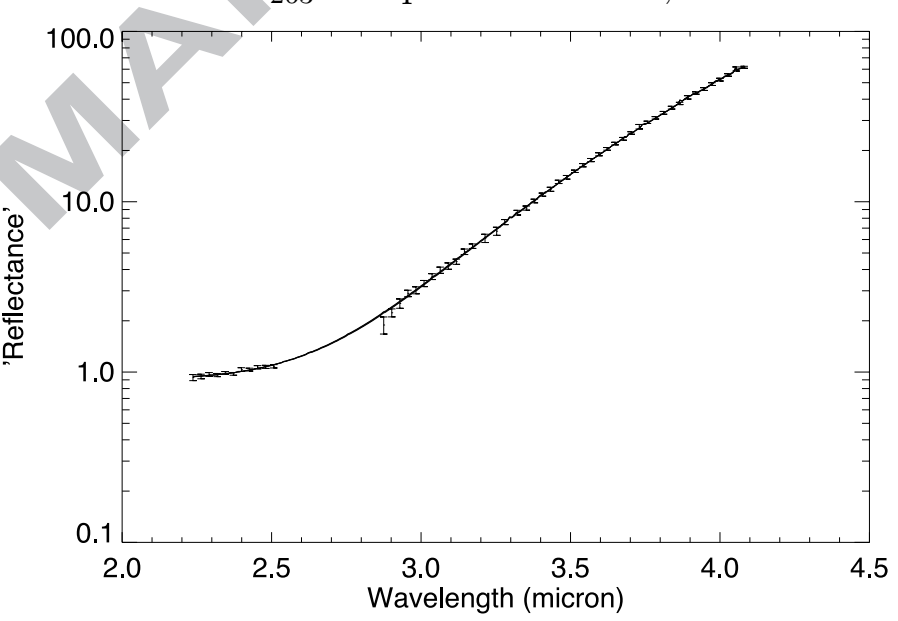

Fig. 1. Thermal-model fits to the measured spectrophotometry and spectroscopy of the asteroid (5381) Sekhmet, (164121) $2003 \mathrm{YT}_{1}$, and (153591) $2001 \mathrm{SN}_{263}$. The curve in each plot represents the near-Earth asteroid thermal model. The model curves are best fits generated by finding the values of diameter, albedo $\eta$ that minimize $\sum_{N}\left[\left(F_{n}(o b s)-F_{n}(\operatorname{model})\right) / \sigma_{n}\right]^{2}$, where $\sigma_{n}$ are the statistical uncertainties in the photometry, $F_{n}(o b s)$. In the case of $2001 \mathrm{SN}_{263}$ (lower right panel), spectroscopic rather than spectrophotometric data were used at much shorter wavelengths. See text for details. Note that the error bars reflect only the statistical uncertainties in the flux derivation from the synthetic aperture procedure. Lightcurve uncertainties, absolute calibration uncertainties, and varying atmospheric transmission contribute to the scatter of the data. 


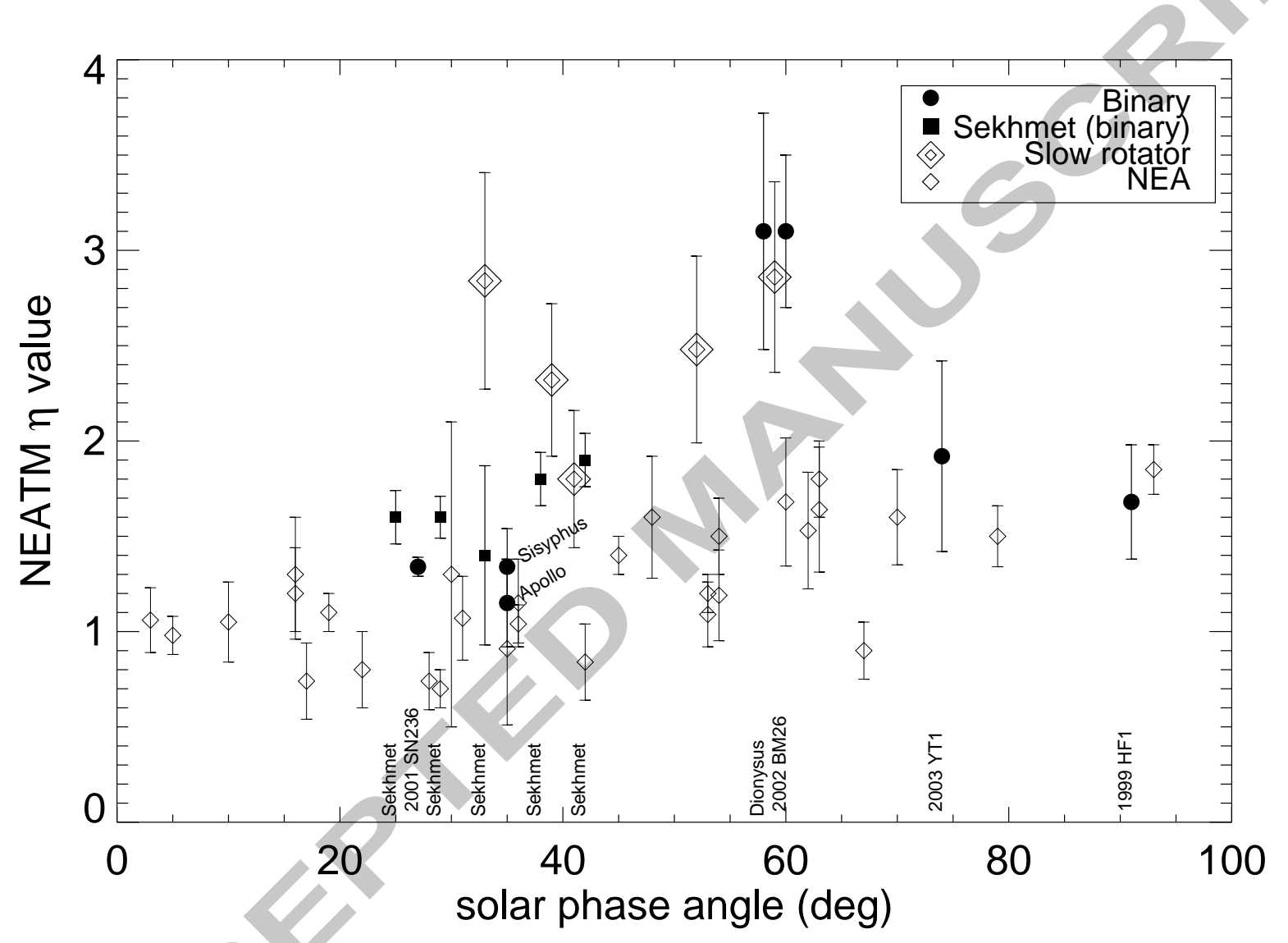

Fig. 2. Dependence of measured $\eta$ values on phase angle, $\alpha$. Objects observed at more than one phase angle appear multiple times. Where available, error bars are taken from the original works, otherwise a conservative uncertainty of $20 \%$ is assumed. Open diamonds: Non-binary NEAs with rotation periods < 10 hours. Double open diamonds: Non binary, slow-rotator NEAs with rotation periods $>10$ hours. Filled symbols: binary NEAs. Filled squares: different observations of the same object: (5381) Sekhmet. 


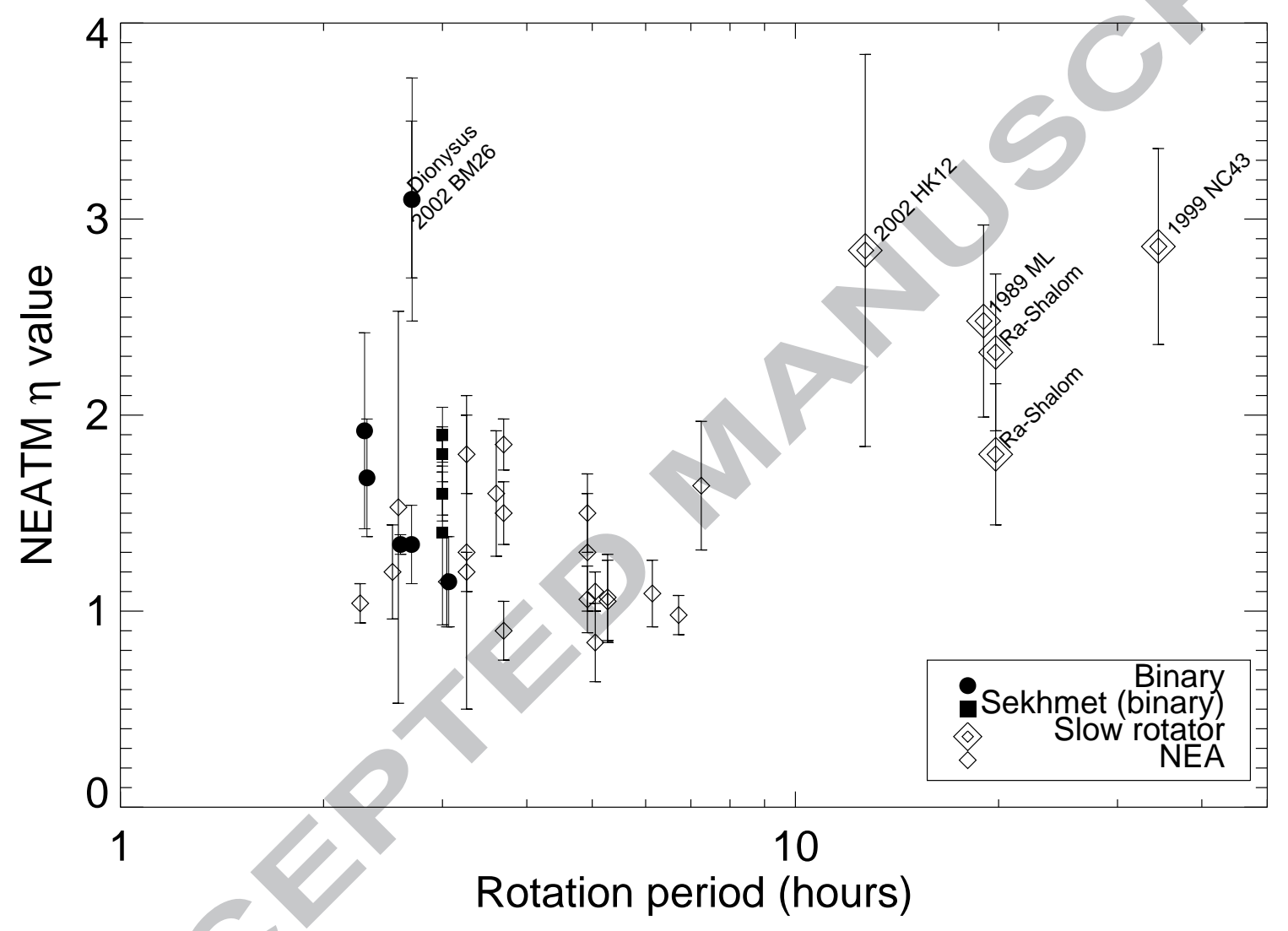

Fig. 3. Dependence of $\eta$ values on rotation period. Open diamonds: Non-binary NEAs with rotation periods $<10$ hours. Double open diamonds: Non binary, slow rotator NEAs with rotation periods $>10$ hours. Filled symbols: binary NEAs. Filled squares: different observations of the same object: (5381) Sekhmet. 


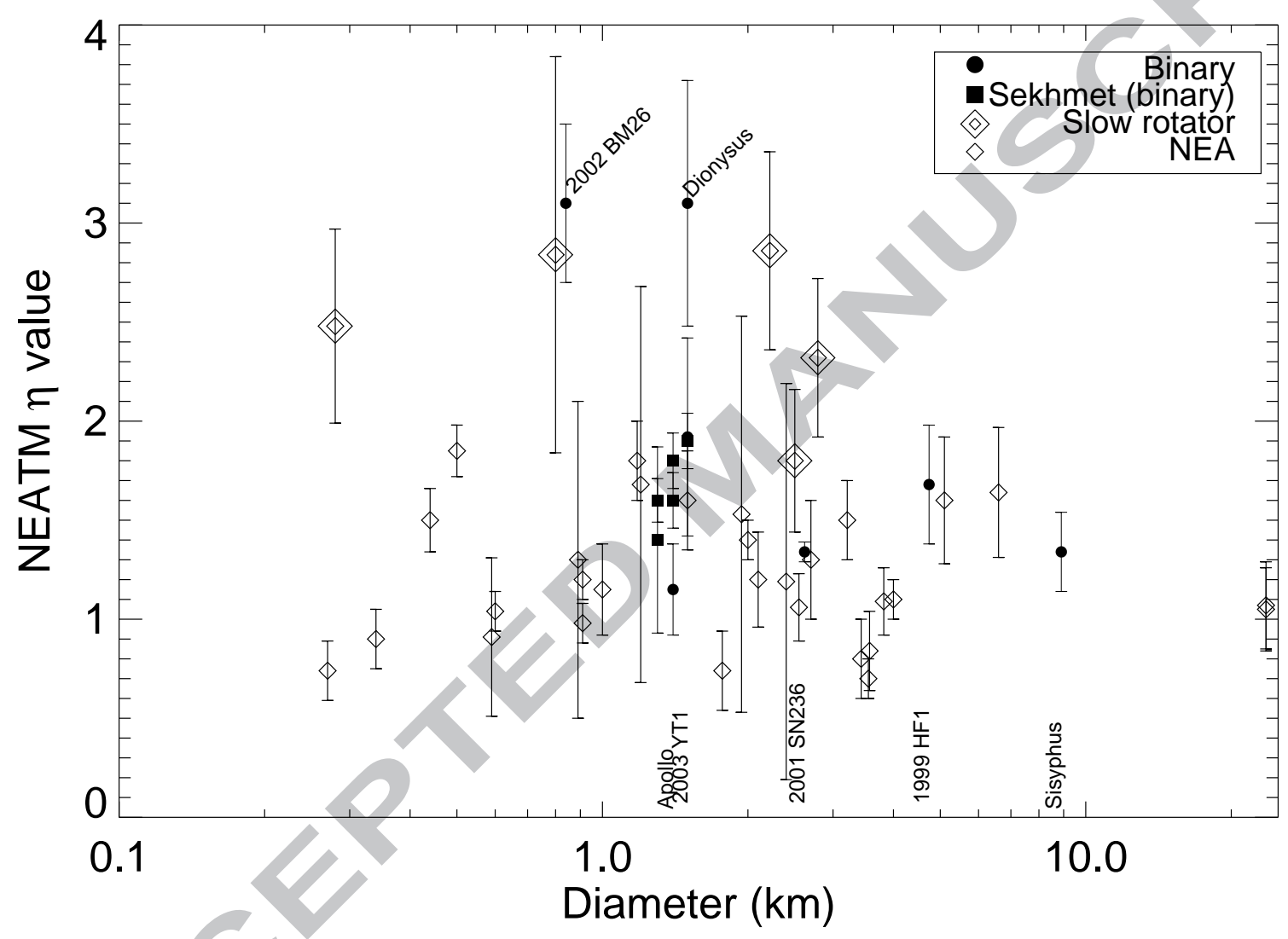

Fig. 4. Dependence of $\eta$ values on asteroid size. Open diamonds: Non-binary NEAs with rotation periods $<10$ hours. Double open diamonds: Non binary, slow rotator NEAs with rotation periods $>10$ hours. Filled symbols: binary NEAs. Filled squares: different observations of the same object: (5381) Sekhmet. 


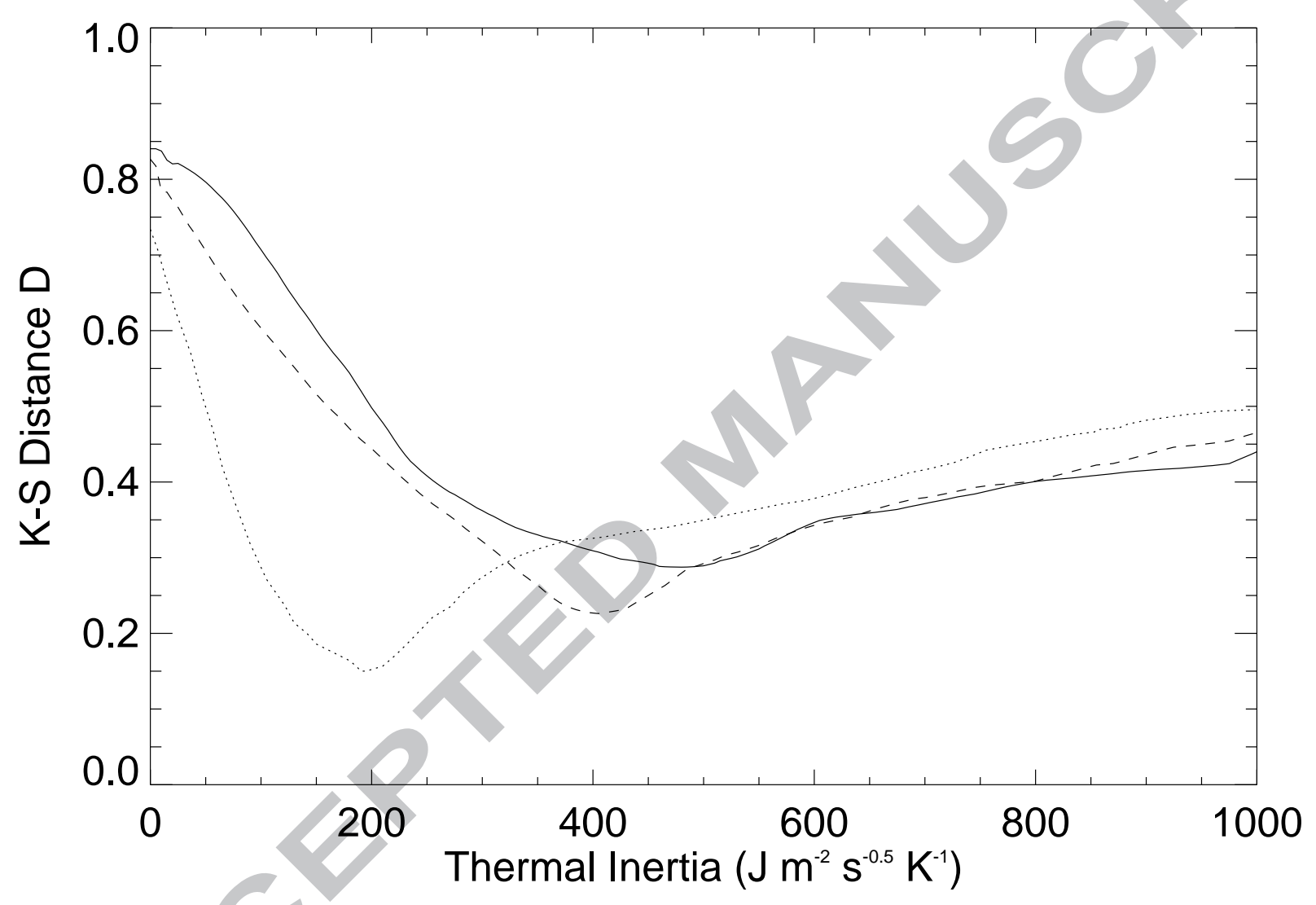

Fig. 5. Kolmogorov-Smirnov distance $D(\Gamma)$ (see text, Sect. 4 for details) as a function of thermal inertia $\Gamma$. Continuous line: binary NEAs, nominal case; dashed line: binary NEAs, fully polarized spin-vector distribution $\left(\theta_{\mathrm{SS}}=0^{\circ}\right)$; dotted line: all NEAs from Delbo' et al. (2007). 
- Binary near-Earth asteroids (NEAs) have higher thermal inertia than typical NEAs.

- Slow-rotator NEAs also have higher thermal inertia than typical NEAs.

- Implication: relative lack of fine regolith on binary and slow-rotator NEAs.

- Implication: a binary formation process involving regolith movement.

- This supports formation of binary NEAs through YORP-induced disruption. 